\title{
Multiple esophageal submucosal hematomas
}

\author{
Vincent Zimmer MD, Frank Lammert MD
}

Previously published at www.cmaj.ca

$\mathrm{A}$ 68-year-old woman presented with an episode of painless hematemesis that later progressed to retrosternal pain radiating to the epigastric area. She was taking acetylsalicylic acid as prophylaxis for cerebrovascular disease. The patient was hemodynamically stable, but her hemoglobin concentration was 81 (normal 120-150) g/L. After myocardial ischemia was excluded, an esophagogastroduodenoscopy showed multiple, sharply demarcated red lesions of varying sizes in the distal esophagus and large amounts of blood in the stomach (Figure 1A).

A repeat esophagogastroduodenoscopy performed the next day showed a small ulcer in the gastric body that had been missed during the first examination. Although some of the esophageal lesions remained unchanged, others had become areas of sloughed mucosa, consistent with spontaneous luminal rupture. The patient was given high-dose acid suppression therapy by continuous intravenous administration of pantoprazole $8 \mathrm{mg} / \mathrm{h}$ and transfused two units of packed red blood cells. The patient recovered uneventfully and her chest pain gradually subsided. A repeat endoscopy performed eight days later showed complete resolution of the esophageal lesions (Figure 1B).

Esophageal submucosal hematoma is an uncommon condition in the spectrum of esophageal injury ranging from mucosal tears (Mallory-Weiss syndrome) to transmural perforation (Boerhaave syndrome). Elderly patients who have intrinsic coagulopathies or are taking anticoagulant or antiplatelet medication are at higher risk for the condition. Patients may present with chest pain, dysphagia or hematemesis. Esophageal hematomas are usually secondary to traumatic injury caused by vigorous vomiting, ingestion of a toxic substance or foreign $\approx$ body, or complications of esophageal instrumentation. ${ }^{1}$ Our patient's hematemesis was not due to esophageal hematoma, but rather to a bleeding ulcer. Acetylsalicylic acid therapy and

From the Department of Medicine II, Saarland University Hospital, Homburg, Germany

CMAJ 2009. DOI:10.1503/cmaj.081683 changes in pressure in the esophageal wall related to vomiting likely contributed to formation of the hematoma.

Although unusual, esophageal hematoma is a potential cause of acute chest pain that may become more common given the increasing use of multiple antithrombotic agents. Early diagnosis can be challenging and relies on adequate imaging studies, such as computed tomography, unless additional symptoms prompt endoscopic evaluation. ${ }^{2}$ If endoscopy shows a large isolated hematoma, radiological exclusion of aortoesophageal fistula is warranted. ${ }^{3}$ Once the diagnosis is established, a conservative approach is usually appropriate because spontaneous luminal rupture followed by uncomplicated mucosal healing commonly occurs. ${ }^{4}$

This article has been peer reviewed.

Competing interests: None declared.

\section{REFERENCES}

1. Ouatu-Lascar R, Bharadhwaj G, Triadafilopoulos G. Endoscopic appearance of esophageal hematomas. World J Gastroenterol 2000;6:307-9.

2. Restrepo CS, Lemos DF, Ocazionez D, et al. Intramural hematoma of the esophagus: a pictorial essay. Emerg Radiol 2008;15:13-22.

3. Maher MM, Murphy J, Dervan P, et al. Aorto-oesophageal fistula presenting as a submucosal oesophageal haematoma. Br J Radiol 1998;71:972-4.

4. Nagai T, Torishima R, Nakashima H, et al. Spontaneous esophageal submucosal hematoma in which the course could be observed endoscopically. Intern Med 2004;43:461-7. 